\title{
The Microbial Diversity of Cabbage Pest Delia radicum Across Multiple Life Stages
}

Tijs J. M. van den Bosch and Cornelia U. Welte*

Department of Microbiology, Institute for Water and Wetland Research, Radboud University, Nijmegen, Netherlands

The cabbage root fly Delia radicum is a worldwide pest that causes yield losses of many common cabbage crops. The bacteria associated with $D$. radicum are suggested to influence the pest status of their host. In this study, we characterized insectassociated bacteria of $D$. radicum across multiple life stages and of their diet plant (turnip, Brassica rapa subsp. rapa) by sequencing the V3-V4 region of $16 \mathrm{~S}$ rRNA genes using the Illumina MiSeq platform. In total, over 1.2M paired-end reads were obtained, identifying 1006 bacterial amplicon sequence variants (ASVs) in samples obtained from the eggs, larvae, pupae and adults of $D$. radicum, as well as turnips that were either fresh or infested with $D$. radicum larvae. The microbial community in $D$. radicum was dominated by Wolbachia, a common endosymbiont of arthropods which we found in all of the investigated insect samples, with the pupal stage having the highest relative abundance. Moderate amounts of Firmicutes were found only in adult $D$. radicum flies, but not in previous life stages. Actinobacteria were mostly found on the eggs and on the skin of fresh plants on which the eggs were deposited. These plants also harbored a large amount of Pseudomonas. The bacterial diversity of the healthy turnip was low, whereas the microbial community of decaying turnips that were heavily infested by $D$. radicum larvae and showing symptoms of advanced soft rot was characterized by a high bacterial diversity. Taken together, this work provides insights into the bacterial communities associated with the cabbage pest $D$. radicum and its associated disease symptoms.

Keywords: 16S rRNA sequencing, microbial diversity, cabbage root fly, Delia radicum, community profiling, DADA2, phyloseq

\section{INTRODUCTION}

Studies that characterize the microbial community profiles of different insect species are gaining increasing attention, yet not much is known about the microbiome of the cabbage root fly Delia radicum. This crop pest can cause devastating yield losses on many popular vegetable crops, e.g., cabbage, kohlrabi or rapeseed (Soroka et al., 2004; Dosdall et al., 2012). The females of this species deposit their eggs onto or near the stem of cruciferous plants and emerging larvae bore into root tissue. The larvae generally stay inside the plant tissue, only to move into the soil just before pupation and to subsequently emerge as adults (Smith, 1927). Metamorphosis causes drastic changes in the anatomy and physiology of the insects, and studies on other insects are reporting 
that it can also have drastic effects on the associated microbiome (Geib et al., 2009; Hammer et al., 2014; Hammer and Moran, 2019).

The importance of symbiotic bacteria has long been well established in a multitude of Eukaryotic models. Also in insects, symbionts have been shown to have important functions in host physiology, e.g., by supplying essential amino acids, conferring resistance against insecticides, or even preventing predation by other insects (Buchner, 1965; Oliver et al., 2005; Kikuchi et al., 2012). The majority of the associations between insect guts and bacteria are of a facultative nature and appear to perform contextdependent functions (Mason et al., 2018). Gut microbes are thus suggested to mediate insect interactions with plant defensive compounds, effectively improving herbivore performance in real time, and between generations (Mason et al., 2018). Diet is an important factor in determining the fitness of any insect, and is a factor that can easily be manipulated in laboratory cultures. A near-aseptic laboratory culture of the diamondback moth (Plutella xylostella) showed increased fitness after being treated with antibiotics and then inoculated with symbiotic bacteria that were resistant to that antibiotic (Somerville et al., 2019). Although this study showed that certain gut symbionts of $P$. xylostella can have significant impact on fitness, the intestinal tracts of caterpillars are typically characterized by short transit times and a high $\mathrm{pH}$ which make microbial colonization difficult, and wild Lepidoptera (caterpillars) have been suggested to typically lack resident gut symbionts (Hammer et al., 2017).

Regarding the microbiome of $D$. radicum, a comprehensive analysis of the gut-associated microbial community associated to different developmental life stages compared to the healthy and invaded feed plant is lacking. A 2006 study by Lukwinski and co-workers (Lukwinski et al., 2006) was the first to analyze the gut microbial community of eggs, larval midgut and feces using a culturing approach. They found that the culturable midgut microbial community was primarily composed of Gammaproteobacteria. The microbial communities of eggs and fecal material were only analyzed regarding their colony forming units, and no further identification was performed. Subsequently, Welte et al. (2016a) performed a metagenome analysis of the larval gut microbial community which provided a more detailed view, largely congruent with the findings by Lukwinski et al. (2006), showing Gammaproteobacteria as the most abundant group of gut-associated microorganisms and several others (Wolbachia, Bacteroidetes, Firmicutes, Actinobacteria, Betaproteobacteria) in lower abundance. Bili et al. (2016) analyzed the microbial communities associated to adult flies of two different populations of $D$. radicum. They found that both D. radicum populations showed Wolbachia as most dominant associated microbial group. Both populations also harbored a low amount of Firmicutes ( $\leq 1 \%$ abundance), and one of the two populations' microbiomes showed an abundant fraction of Gluconacetobacter (18\% abundance).

In this study, our objective was to compare the microbial communities associated to the different life stages of $D$. radicum as well as healthy and invaded food sources. We profiled the microbial community of the eggs, larvae, pupae and adults. We also identified the major differences of bacterial communities that were associated with healthy the feed plants Brassica rapa subsp. rapa pre-predation, compared to plants that were macerated after being invaded by $D$. radicum larvae. This rot only developed in turnips that were used as egg laying substrate by $D$. radicum, suggesting that it is an effect caused either directly by the larvae or by larva-associated bacteria, which is a mechanism that is well described in Drosophila (Blum et al., 2013). Bacterial isolates from $D$. radicum have already shown the potential for metabolizing secondary metabolites that are associated with the diet plant of D. radicum (Welte et al., 2016a). Our results lead to the description of the microbial communities of multiple life stages of $D$. radicum as well as that of the environment that is created in the diet plant of the larvae. This work provides novel data for the research of symbioses in largely unstudied holometabolous insect groups, and may ultimately even aid in identifying transmission routes of insect and plant-associated bacteria as well as potential novel targets in microbial pest control.

\section{MATERIALS AND METHODS}

\section{Delia radicum Rearing}

The eggs that formed the starting colony of $D$. radicum were obtained from Wageningen University (Laboratory of Entomology, Prof. Joop van Loon). Flies were kept in an entomology cage $(60 \times 60 \times 120 \mathrm{~cm})$ with ad libitum access to water and a 1:1:1 mixture of dry food consisting of yeast extract, skim milk powder and sucrose. Cages were kept in a laboratory without humidity control at room temperature and under natural lighting. For breeding purposes, a small plastic container $(12 \times 12 \times 6 \mathrm{~cm}$, now referred to as egg box $)$ was filled with $1 \mathrm{~cm}$ of wetted river sand upon which a turnip (B. rapa subsp. rapa) was placed to facilitate egg deposition. Egg boxes were moved out of the entomology cage after a week and the larvae were left to feed on the supplied turnip for approximately 4 weeks until pupation. Pupae were then separated from the river sand by flooding the egg box and sieving the water, after which the pupae were placed back into an entomology cage until eclosion, marking the beginning of a new generation.

\section{Sample Acquisition \\ Turnip (TURN1-5)}

Skin of turnips (B. rapa subsp. rapa) was cut superficially with a surface-sterilized razor blade, approximately $2 \mathrm{~mm}$ thick. Approximately $5 \mathrm{~g}$ of this material was homogenized in liquid nitrogen by the use of mortar and pestle. Approximately $0.25 \mathrm{~g}$ of homogenous material was used downstream with PowerSoil DNA extraction.

\section{Decaying Turnip Tissue (PULP1-5)}

Approximately $200 \mathrm{mg}$ samples of macerated tissue from a D. radicum maggot-infested turnip were transferred directly to a PowerSoil tube with a surface-sterile spatula.

\section{Eggs (EGGS1-5)}

Approximately 200 D. radicum eggs were collected by flooding the egg boxes with (non-sterilized) demiwater, and collecting the 
runoff in a thin-necked volumetric flask in order to concentrate the floating eggs at the top of the flask. They were then collected with a Whatman filter, and placed in a filter holder. Another Whatman filter was placed on top and the eggs were washed with $5 \mathrm{~mL}$ of the following solutions using a syringe: milliQ water, $6.5 \%$ bleach, $70 \%$ ethanol, and milliQ water. Subsequently, the Whatman filters containing the eggs were homogenized by freezing in liquid nitrogen and then crushing by mortar and pestle. The homogenate was used downstream in DNA extraction using the PowerSoil kit.

\section{Larvae (MAGG1-10)}

Twenty larvae were collected with sterile forceps and stored at $-20^{\circ} \mathrm{C}$ until DNA extraction. Prior to DNA extraction, all were washed in $5 \mathrm{~mL}$ sterile MQ water, then in $5 \mathrm{~mL} \mathrm{70 \%} \mathrm{ethanol,}$ then in 5.5-7.5\% active chlorine bleach, and lastly rinsed in $5 \mathrm{~mL}$ MQ again. Subsequently, all whole larvae were homogenized by mortar and pestle in liquid nitrogen.

\section{Pupae (PUPA1-5)}

40-100 pupae were collected by flooding an egg box with tap water, and running the top liquid containing the pupae through a generic sieve. Prior to DNA extraction, all pupae were surface-sterilized in an identical fashion to the larval samples. Subsequently, all whole pupae were homogenized by mortar and pestle in liquid nitrogen.

\section{Flies}

30-40 flies were collected from entomology cages with an aspirator and subsequently killed with chloroform. Flies were sexed based on the morphology of the abdomen; flies with swollen abdomens that are characteristic for gravid females (FLYF1-5) were designated as females and flies with shriveled abdomens (FLYM1-5) were designated as males. Individuals with inconclusive exteriors were discarded. Flies were surfacesterilized in an identical fashion to the larval samples and subsequently homogenized by mortar and pestle in liquid nitrogen. For detailed descriptions on corresponding origins and amounts of biological material that was used per sample, as well as the DNA concentrations after extraction, we refer to the metadata table (Supplementary Table 1).

\section{Sequencing and Analysis of the $16 \mathrm{~S}$ rRNA Gene V3-V4 Region}

After sample preparation, all 40 samples were weighed and DNA was extracted using the DNeasy PowerSoil kit (Qiagen) according to the manufacturer's protocol. Due to low DNA concentrations, the samples were concentrated using a SpeedVac Vacuum concentrator. Samples were submitted to BaseClear (Leiden, the Netherlands) for paired-end sequencing of the V3-V4 region on the Illumina MiSeq system, where the primers CCTACGGGNGGCWGCAG and GACTACHVGGGTATCTAATCC were used for the generation of the V3-V4 region amplicon (Klindworth et al., 2013). 80 paired-end FASTQ read sequence files (two per sample) were generated using bcl2fastq2 version 2.18 and initial quality assessment was based on data passing the Illumina Chastity filtering. Reads containing PhiX control signal were removed using an in-house filtering protocol by BaseClear. Second quality assessment was based on the remaining reads using the FASTQC quality control tool version 0.11.5. Raw reads were delivered demultiplexed and without non-biological nucleotides (i.e., sequencing primers, adapters, linkers), and the primers used for amplification of the V3-V4 region were manually removed by deleting the first 17 NTs from forward reads and the first 21 NTs from reverse reads using simple unix commands prior to preprocessing. Preprocessing of the sequencing data was done using the DADA2 pipeline (Callahan et al., 2016). Taxonomic assignment of the reads was done up to the species level with DADA2 using the Silva non-redundant database version 128 (Yilmaz et al., 2014). A phylogenetic tree of the 1006 ASVs that were resolved by the DADA2 pipeline was created by RAxML (Stamatakis, 2006). Data visualization and analysis were performed using the phyloseq package (McMurdie and Holmes, 2013). Chaol was used for a measure of estimated richness, whereas the Shannon-Weaver index provides more information about community composition and evenness by considering relative abundances (Kim et al., 2017). In order to compare sample groups and test the null hypothesis that the dispersion of the groups as defined by measure space are equivalent for all groups, we performed permutational multivariate analysis of variance (PERMANOVA) using adonis from the 'vegan' package in R (Oksanen et al., 2019). Distance matrices were built with the Bray-Curtis method of vegdist and multilevel pairwise comparisons were performed in 999 permuations with a wrapper for adonis (Martinez Arbizu, 2019).

\section{RESULTS}

\section{Preprocessing}

Paired-end sequencing of the V3-V4 region of the 16S rRNA gene resulted in a total of 1,249,018 reads from 40 samples. Quality score profiles were typical for MiSeq sequencing, with high quality scores for $>250$ cycles in the forward direction and $\sim 200$ cycles in the reverse direction (Supplementary Figure 1). Rarefaction curves indicated that the coverage of the bacterial diversity was sufficient and we therefore chose not to subsample to an equal depth per sample. Recently developed methods allowed us to resolve amplicon sequence variants (ASVs) instead of resorting to the more classical construction of molecular operational taxonomic units (OTUs). The benefits of this method include higher resolution and reproducibility, and allows for simple merging between independently processed datasets (Callahan et al., 2017). Unless stated otherwise, the data and analyzes of this manuscript excludes reads that were taxonomically assigned to mitochondria, chloroplast, or where the taxonomic assignment was not resolved at the phylum level.

\section{Bacterial Community Composition}

A total of 1006 amplicon sequence variants (ASVs) were identified in the entire dataset. Filtering ASVs with a relative abundance mean smaller than $5 \mathrm{e}-5$ left a total of 357 abundant ASVs across ten phyla (Figure 1A). All D. radicum samples, 

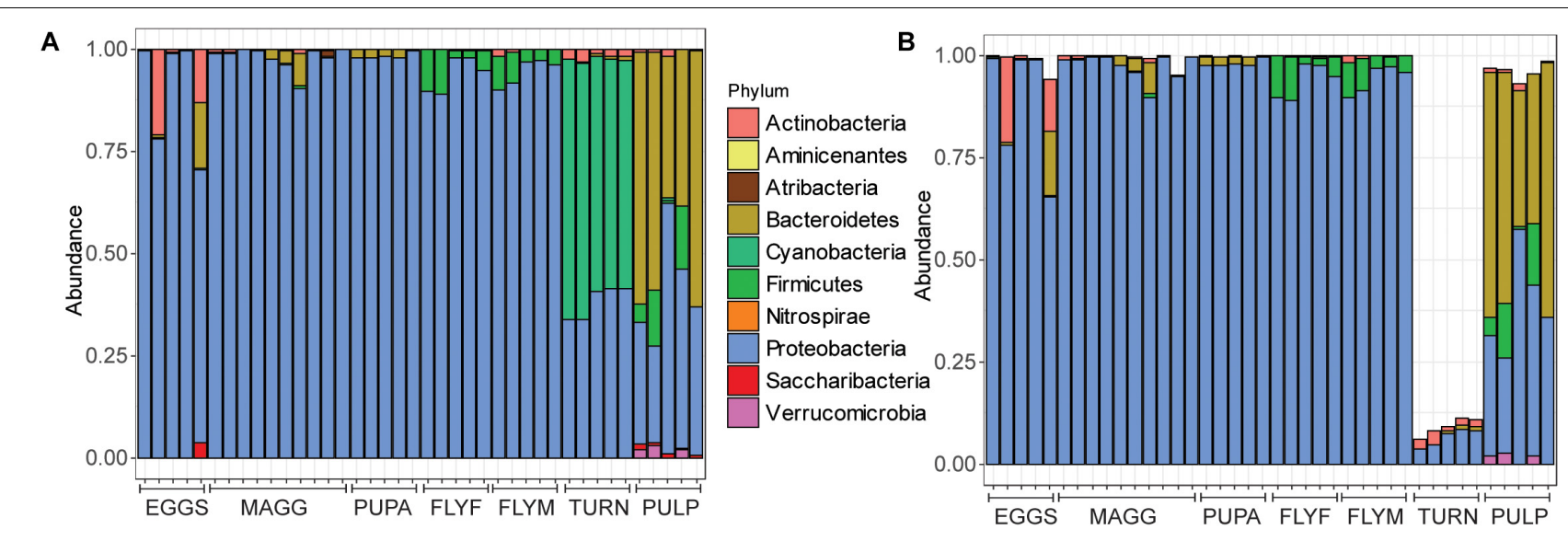

C
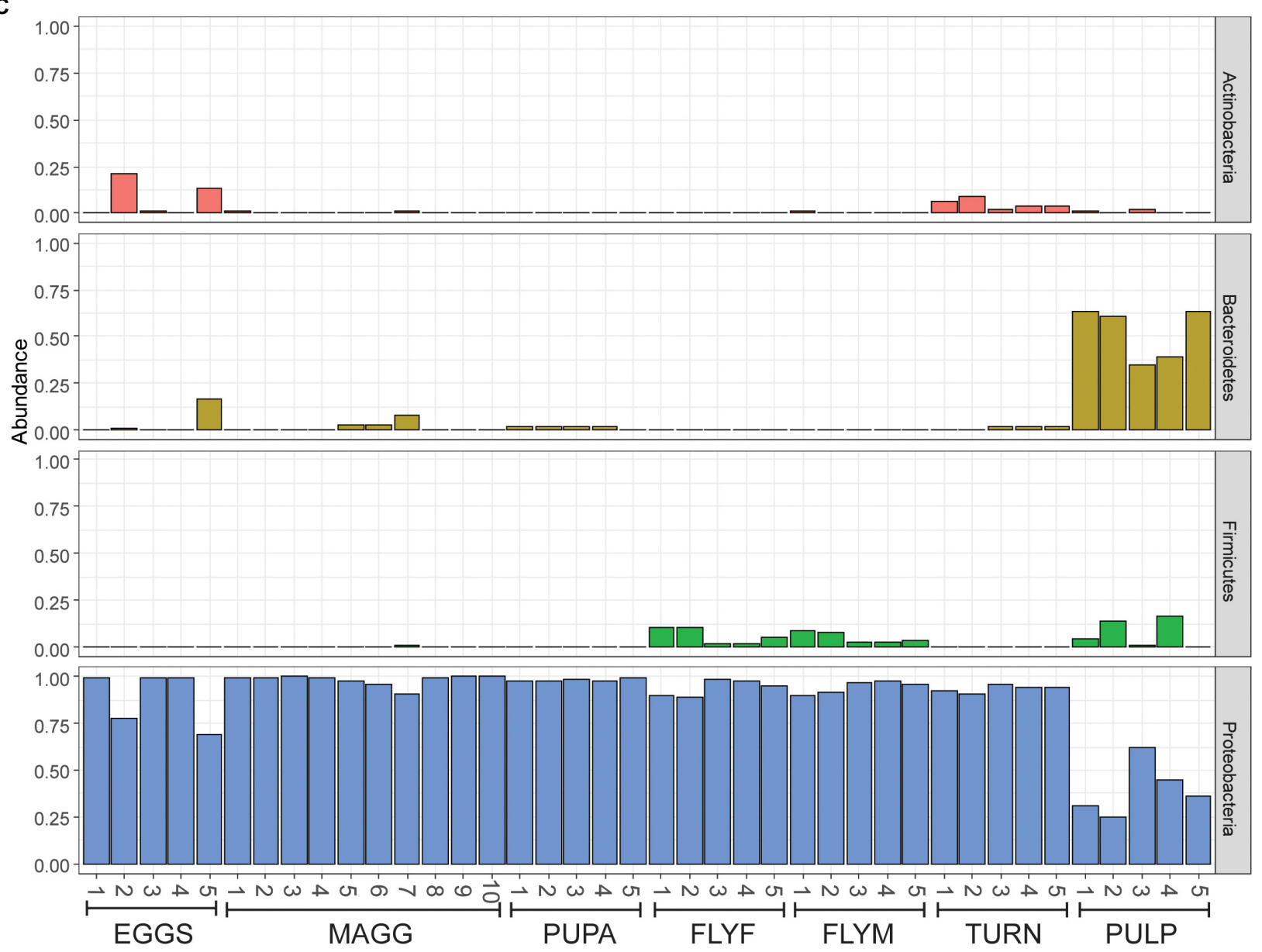

FIGURE 1 | (A) All reads represented as relative abundance, after filtering of low-abundance reads $($ mean $(x)>5 e-5)$. (B) Bar plot after removal of reads where Class was designated as "Chloroplast" or "Unidentified," or where Family was designated as "Mitochondria." Note that over $80 \%$ of reads were removed from samples in the "turnip" sample group, which can be explained by the high number of chloroplasts within the tissue. (C) Relative reads of Actinobacteria, Bacteroidetes, Firmicutes, and Proteobacteria in all samples, faceted by phylum. EGGS, Delia radicum eggs; MAGG, Delia radicum larvae; PUPA, D. radicum pupae; FLYF, Gravid female adults of $D$. radicum; FLYM, Male adults of $D$. radicum; TURN, Skin of fresh, non-infested Brassica rapa subsp. rapa; PULP, Macerated tissue of a $D$. radicum-infested individual of $B$. rapa subsp. rapa.

regardless of life stage, were largely dominated by Proteobacteria. Most of the sequence data gathered from the samples of intact turnips $(\sim 90 \%)$ consisted of ASVs that came from chloroplasts and were thus not suitable for the determination of its microbiome (Figure 1B). Moderate amounts of Firmicutes appeared in adult $D$. radicum flies, but not in previous life 
stages (Figure 1C). The remaining turnip ASVs were mostly classified as Proteobacteria and Actinobacteria. Samples of decaying turnip tissue contained high numbers of Bacteroidetes as well as Proteobacteria (Figure 1C), and uniquely harbored Verrucomicrobia ASVs (not shown). Contamination from chloroplast was not apparent in these samples. An overview of the 20 most abundant genera across all samples is presented in Figure 2A, and a heatmap depicting the relative abundances of different bacterial families across sample groups can be seen in Figure 2B.

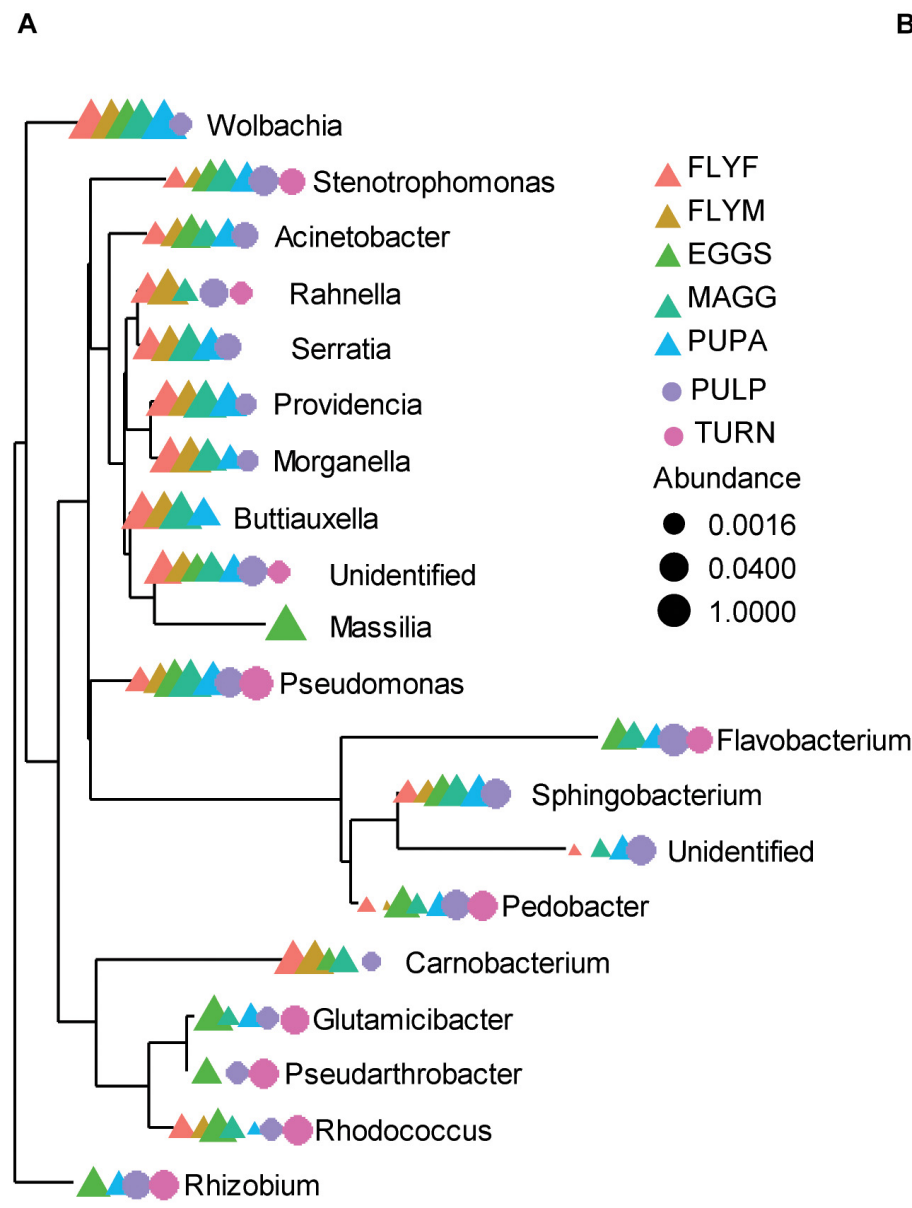

B Verrucomicrobiaceae Anaplasmataceae Xanthomonadaceae Moraxellaceae Shewanellaceae Enterobacteriaceae Oxalobacteraceae Comamonadaceae Alcaligenaceae Methy lophilaceae Chromatiaceae Pseudomonadaceae Cellv ibrionaceae Bacteriov oracaceae Rhodobacteraceae Sphingomonadaceae Hy phomonadaceae Hy phomicrobiaceae Rhizobiaceae Brucellaceae Caulobacteraceae Brady rhizobiaceae Methy lobacteriaceae

Nitrospiracea $\gtrsim \quad$ Veillonellaceae $\frac{c}{\sigma} \quad$ Bacillaceae $\stackrel{\sim}{\sim}$ Leuconostocaceae Lactobacillaceae

Enterococcaceae Carnobacteriaceae Staphy lococcaceae Paenibacillaceae Clostridiaceae 1 Lachnospiraceāe

Flav obacteriaceae Sphingobacteriaceae Porphy romonadaceae Chitinophagaceae Cy tophagaceae Cry omorphaceae Micrococcaceae Microbacteriaceae Demequinaceae Abundance $4.0000000000-$
$0.0625000000^{-}$
$0.0009765625-$

Beutenbergiaceae Sanguibacteraceae Cellulomonadaceae Intrasporangiaceae Nocardiaceae Tsukamurellaceae Cory nebacteriaceae Nocardioidaceae Propionibacteriaceae

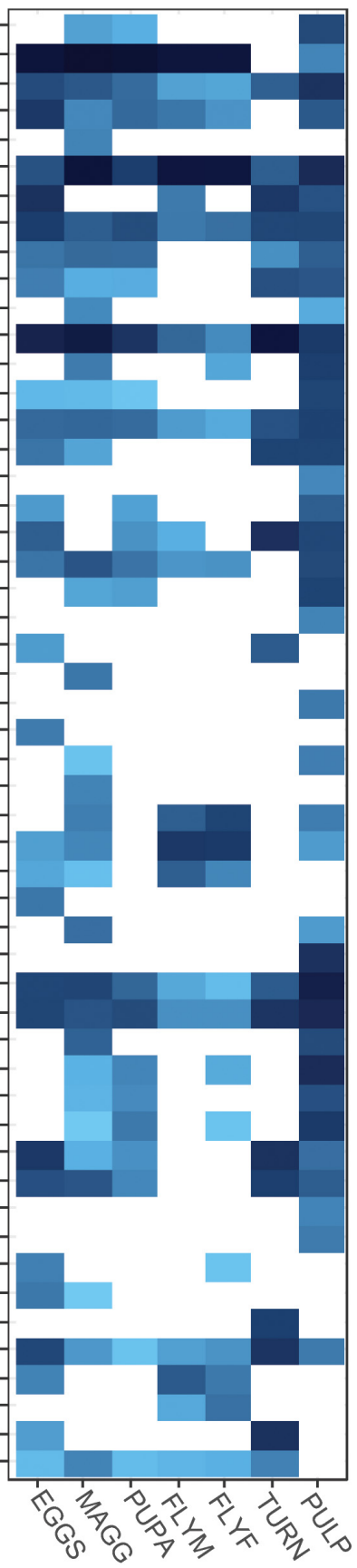

FIGURE 2 | (A) Overview of the 20 most abundant genera associated with a laboratory culture of Delia radicum including samples from multiple life stages, and from fresh and decayed turnips. Data was first agglomerated at the genus level, then merged per sample. (B) Heatmap that shows the relative abundance of reads per sample groups, where abundances were merged at the Family level. EGGS, D. radicum eggs; MAGG, D. radicum larvae; PUPA, D. radicum pupae; FLYF, Gravid female adults of $D$. radicum; FLYM, Male adults of $D$. radicum; TURN, Skin of fresh, non-infested Brassica rapa subsp. rapa; PULP, Macerated tissue of a $D$. radicum-infested individual of $B$. rapa subsp. rapa. 


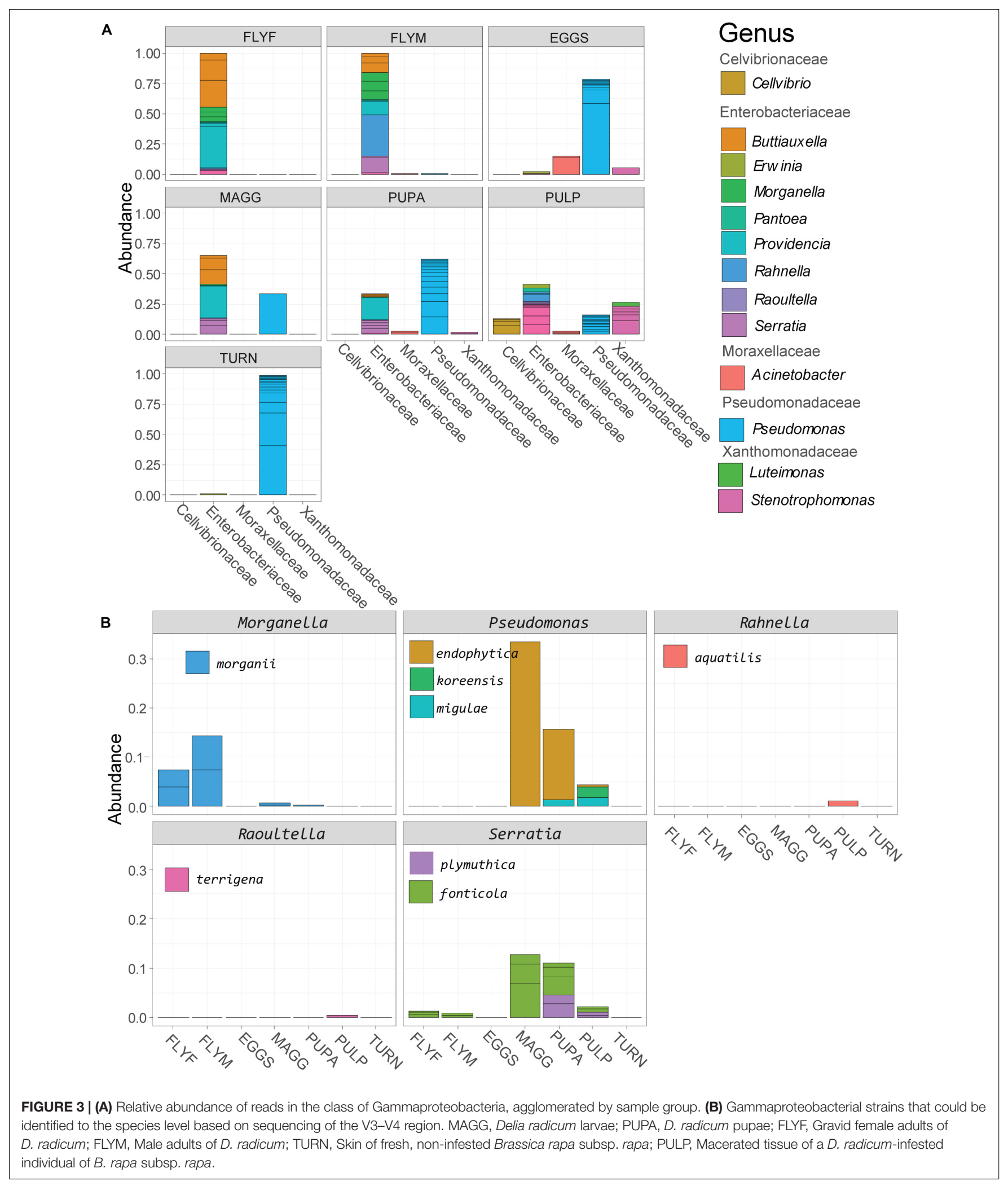

\section{Gammaproteobacteria}

Gammaproteobacterial reads made up $31 \%$ of the reads in the dataset and we decided to use this subset for a deeper analysis (Figure 3A). We found that almost all adult fly ASVs in the Gammaproteobacteria class were affiliated with the family Enterobacteriaceae, whereas larvae and pupae also contained 
considerable amounts (30-62\%) of Pseudomonas reads. High relative abundances $(>75 \%)$ of ASVs in the Pseudomonas genus were found in $D$. radicum egg samples and in fresh turnip skin samples. The Gammaproteobacterial ASVs found in the decayed turnip tissue represented all five families. One of the advantages of generating amplicon sequence variants with the DADA2 pipeline rather than 97\%-identity-based OTU clustering is that this higher-resolution method allows for the taxonomic assignment of species-level and sometimes even strain-level variants. Strain-level variants can be a source of functional diversity and can represent specialists adapted to particular hosts or environments, the identification of which would otherwise be obscured by OTU clustering (Kwong et al., 2017). The taxonomy of 34 ASVs could be determined to the species level, of which 22 were Gammaproteobacteria, and 8 of these were categorized as abundant (relative abundance $>5 \mathrm{e}$ 4) (Figure 3B). Morganella morganii was found primarily in the adult flies and in small amounts in larvae and pupae. Morganella sp. were also found among the 20 most abundant genera (Figure 2A), so it can be concluded that different species of this genus were present in all samples, among which Morganella morganii. The genus Rahnella was also detected among the 20 most abundant genera (Figure 2A) found in all life stages of D. radicum apart from eggs. One ASV identified down to the species level was Rahnella aquatilis that was primarily found in the decayed turnip tissue, but was previously also reported inside the gut of certain species of longicorn beetles in Korea (Park et al., 2007). Three Pseudomonas species (P. endophytica, P. migulae, P. koreensis) and two species of Serratia (S. fonticola and S. plymuthica) could be identified in larvae, pupae and decayed turnip tissue. Representatives of both genera were also found to be very abundant in all life stages of $D$. radicum (Figure 2A) but the methods employed in this study did not allow for deeper taxonomic identification. Although sequencing of the V3-V4 region did not allow strain-level resolution, it is interesting to note that the strain Serratia plymuthica 3Rp8 was previously isolated from the rhizosphere of Brassica napus L. (Adam et al., 2016).

\section{Wolbachia}

The genus Wolbachia comprises endosymbiotic bacteria and is known to infect a wide variety of arthropods and nematodes (Serbus et al., 2008). In our dataset, ASVs that were taxonomically assigned to the genus Wolbachia made up 52\% of the total reads, which includes ten non-insect samples, nine of which were devoid of Wolbachia reads. Since we extracted the DNA of multiple individuals for every $D$. radicum sample, the infection rates of the culture cannot be determined with this data and it is possible that not $100 \%$ of individuals carry Wolbachia. One out of five samples of decaying turnip ("PULP4") yielded 34 reads of Wolbachia, which is most likely due to the accidental inclusion of larval tissue during sampling. A total of nine different ASVs were designated as Wolbachia, one being represented by 478723 reads, whereas the other eight contained only between 2 and 25 reads. In all likelihood, the detection of the latter variants was the result of sequencing errors and/or artifacts in the ASV-calling algorithm of DADA2. Between-sample variation of

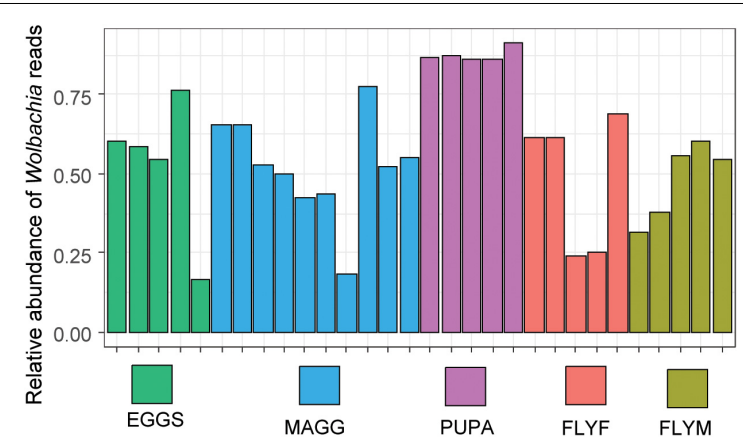

FIGURE 4 | Relative abundance of reads assigned to the genus of Wolbachia in Delia radicum samples. MAGG, D. radicum larvae; PUPA, D. radicum pupae; FLYF, Gravid female adults of $D$. radicum; FLYM, Male adults of D. radicum; TURN, Skin of fresh, non-infested Brassica rapa subsp. rapa; PULP, Macerated tissue of a $D$. radicum-infested individual of $B$. rapa subsp. rapa.

relative abundance of Wolbachia reads in D. radicum life stages is presented in Figure 4.

\section{Sample Diversity}

The complexity within samples (alpha diversity) was analyzed by two diversity metrics (Chaol and Shannon-Weaver) (Figure 5A). Low-abundance reads were included in these analyzes in order to obtain a better indication of sample complexity. The macerated tissue of $D$. radicum-infested turnip showed particularly high complexity by both Chaol and ShannonWeaver indices. Bacterial species richness in the microbiome of D. radicum is highest in the larval stage, most likely due to the presence of a gut that is filled with decaying plant matter that is, as we show here, characterized by a highly complex bacterial community. The Shannon diversity of pupae was particularly low and can be explained by the high abundance of Wolbachia that decreases the evenness of the bacterial community in these samples. The between-sample diversity was visualized by performing principal coordinate analysis (multidimensional scaling), using both weighted and unweighted UniFrac distances (Figure 5B). Assuming a cut-off $p$-value of 0.05 , we found significant differences that explained between $25 \%$ and $71 \%$ of the variation between all sample groups except between the female and male adult fly groups (Table 1). From these results we can conclude that each of the life stages of $D$. radicum has an identifiably unique microbiome. We did not observe significant differences between the microbiome of male and female flies.

\section{DISCUSSION}

In this study we explored the diversity of the microbial community that is associated with the eggs, larvae, pupae and adult life stages of the cabbage root fly $D$. radicum. All developmental life stages contained considerable amounts of Wolbachia reads, but also contained a diverse microbial community of other bacteria. Since diet and environment are also factors that influence the microbiome, we included the 


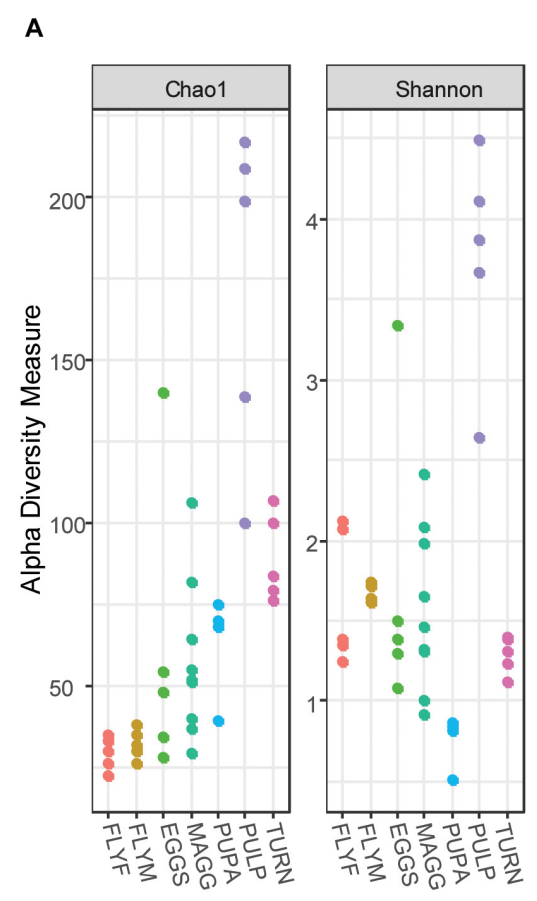

B

PCoA (Weighted UniFrac)
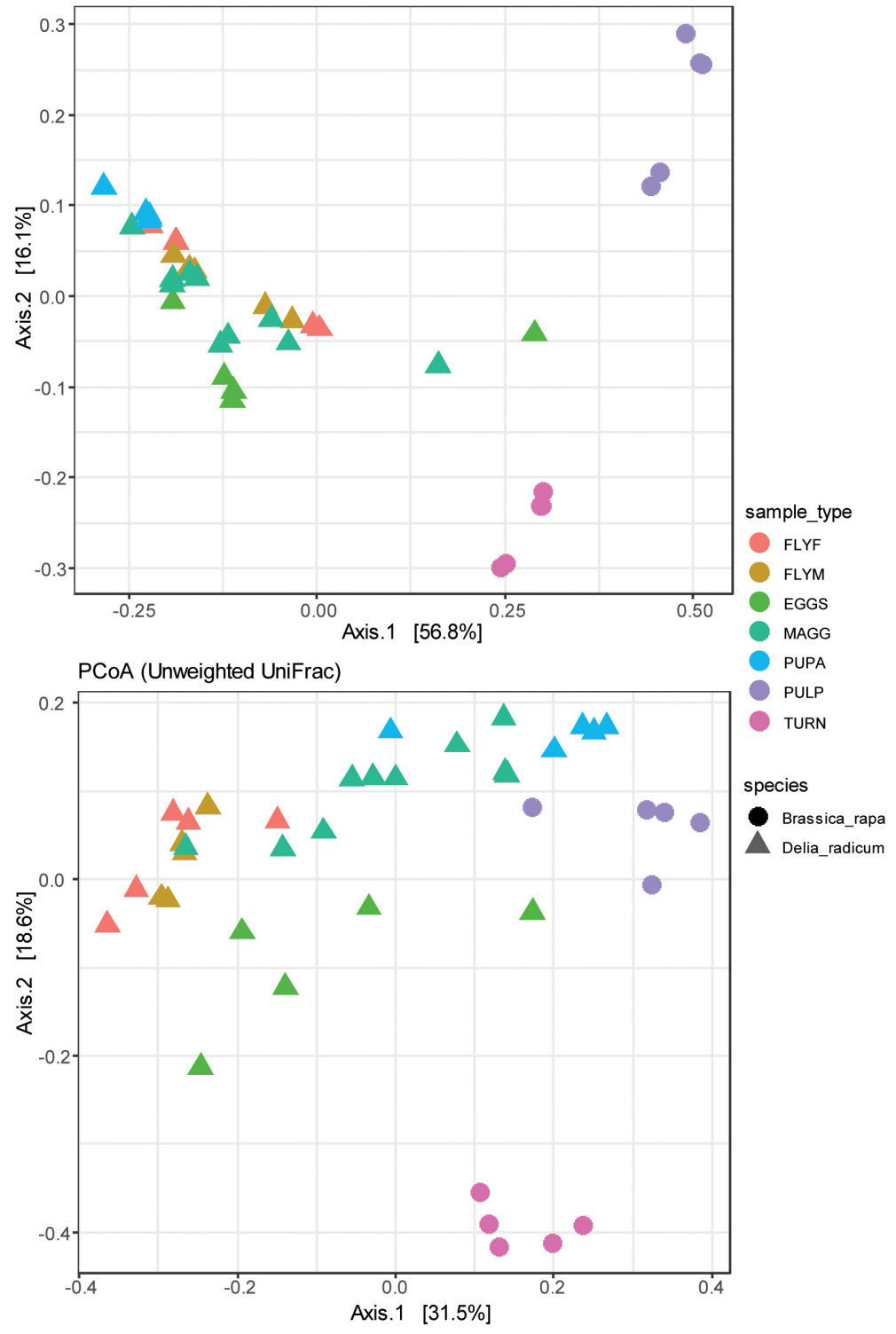

FIGURE 5 | (A) Dot plots of the alpha diversity (Chao1 and Shannon-Weaver indices) of Delia radicum-associated microbiota and their diet substrates pre feeding (turnip_skin) and post feeding by D. radicum larvae (turnip_pulp). (B) Principle coordinate analysis of 30 D. radicum and 10 Brassica rapa samples, based on unweighted and weighted UniFrac distances. MAGG, D. radicum larvae; PUPA, D. radicum pupae; FLYF, Gravid female adults of $D$. radicum; FLYM, Male adults of D. radicum; TURN, Skin of fresh, non-infested B. rapa subsp. rapa; PULP, Macerated tissue of a D. radicum-infested individual of $B$. rapa subsp. rapa.

microbial diversity of the host plant (turnip), and of decayed host plant material that had been infested with $D$. radicum larvae. The decayed plant material was characterized by a very high alpha diversity.

Fresh and macerated turnip samples were furthermore considerably different from the microbial communities of $D$. radicum in structure and composition, but also shared a number of taxa that are possibly transferred between the environment and the host, or vice versa.

Brassica rapa subsp. rapa is a member of the Brassicaceae family of plants which employ a method of defense against herbivores and pathogenic bacteria by producing the secondary metabolite isothiocyanate (Tierens et al., 2001). Our initial expectation was that the high concentration of isothiocyanates within damaged turnip tissue would only allow isothiocyanateresistant bacteria to thrive in this environment. Furthermore, many isothiocyanate-resistant strains are within genera that are also shown to have cell wall degrading properties (Basset et al., 2000; Welte et al., 2016b). Instead of a low diversity of strains that are typical cell wall degraders such as Erwinia, Pectobacterium, or Pantoea, these samples contained a flourishing community of Bacteroidetes and Gammaproteobacteria from all different 
TABLE 1 | Pairwise multilevel comparisons using adonis with 999 permutations.

\begin{tabular}{|c|c|c|c|c|c|c|}
\hline $\operatorname{Pr}(>\mathrm{F})$ & $\begin{array}{l}\text { D. radicum } \\
\text { Eggs }\end{array}$ & $\begin{array}{l}\text { D. radicum } \\
\text { Larvae }\end{array}$ & $\begin{array}{l}\text { D. radicum } \\
\text { Pupae }\end{array}$ & $\begin{array}{c}\text { D. radicum } \\
\text { Adult females }\end{array}$ & $\begin{array}{l}\text { D. radicum } \\
\text { Adult males }\end{array}$ & $\begin{array}{c}\text { Macerated } \\
\text { turnip }\end{array}$ \\
\hline D. radicum Larvae & $0.002^{\star \star}$ & & & & & \\
\hline D. radicum Pupae & $0.009^{\star \star}$ & $0.001^{\star \star \star}$ & & & & \\
\hline D. radicum Adult females & $0.010^{\star \star}$ & $0.007^{\star \star}$ & $0.010^{\star *}$ & & & \\
\hline D. radicum Adult males & $0.016^{\star}$ & $0.001^{\star \star \star}$ & $0.012^{\star}$ & 0.206 & & \\
\hline Macerated turnip & $0.009^{\star \star}$ & $0.001^{* \star \star}$ & $0.012^{*}$ & $0.007^{* \star}$ & $0.011^{\star}$ & \\
\hline Fresh turnip & $0.008^{\star \star}$ & $0.003^{\star \star}$ & $0.010^{\star \star}$ & $0.010^{\star \star}$ & $0.011^{*}$ & $0.009^{\star \star}$ \\
\hline
\end{tabular}

The levels compared are the sample groups that distinguish the Delia radicum life stages. p-values denoted as (0.010-0.05)*, (0.001-0.010)**, or (0-0.001) ${ }^{\star * *}$.

families. Isothiocyanates are volatile and the bioavailability can be expected to differ significantly between the initial moment of $D$. radicum attack and the late-stage infestation marked by complete maceration of the turnip tissue. It is possible that early colonizers are capable of overcoming isothiocyanate-based defenses of the plant, and that a multitude of opportunistic taxa colonize the tissue after the plant defenses have sufficiently diminished over time. The Serratia and Pseudomonas species that were identified in larval samples could potentially be of great nutritional aid for their hosts. Serratia plymuthica 3Rp8, isolated from the rhizosphere of Brassica napus L. (Adam et al., 2016), for example, contains two copies of the saxA gene (Van den Bosch et al., 2018) that has been implied in overcoming isothiocyanate-based plant defenses in plant pathogenic bacteria (van den Bosch et al., 2019).

All life stages of $D$. radicum were characterized by high abundances of Proteobacterial symbionts, but each was shown to have unique characteristics that could be resolved by comparing various diversity metrics.

Comparisons with other studies that sequenced the 16S rRNA gene of insect-associated gut bacteria show some interesting parallels, as well as dissimilarities.

Bili et al. (2016) previously analyzed the bacterial community of $D$. radicum adults of three different geographic locations in France using 454 pyrosequencing of the V4-V5 region of the bacterial 16S rRNA gene (2016). They reported a relatively lowcomplexity microbiome dominated by Wolbachia or Wolbachia and Gluconacetobacter, depending on the geographical origin of the flies. The abundance of Firmicutes was low in the fly samples of their study, aligning well with the data presented here. In contrast to the study performed by Bili et al. (2016), we found high abundances of Enterobacteriaceae and we were able to identify multiple Gammaproteobacterial reads which had not been reported previously. Morganella morganii is found as a resident of the gut microbiome of the common house fly Musca domestica (Gupta et al., 2012) and in the microbiome of healthy bees (Erban et al., 2017) but is also designated as a lethal pathogen in Mexican fruit flies [Anastrepha ludens (Loew)] and sand flies [Lutzomyia longipalpis (Lutz and Neiva)] (Salas et al., 2017). In our dataset, Morganella sp. were abundant in all life stages of D. radicum, most of which could not be assigned to a specific species due to the limitation of the sequencing method. In the oriental fruit fly Bactrocera dorsalis, it was shown that Proteobacteria dominated immature stages, whereas adult stages were dominated by
Firmicutes (Andongma et al., 2015). Although Firmicutes did not quite dominate in $D$. radicum adults, this life stage was the only one where moderate amounts of Firmicute reads were present. In the house fly $M$. domestica, Gammaproteobacteria are dominating larval and adult microbiomes (Gupta et al., 2012; Zhao et al., 2017). Also the microbiomes of other flies harbor a high amount of Gammaproteobacteria, e.g., as found in Drosophila melanogaster (Corby-Harris et al., 2007; Cox and Gilmore, 2007). Furthermore, the genus Comamonas was reported to be the most abundant in pupae and completely absent in adults of $B$. dorsalis (Andongma et al., 2015). This trend was also observed in the D. radicum dataset. $4-6 \%$ of non-Wolbachia reads in all pupae samples were Comamonas, but fly samples contained at most $1 \%$.

For future studies it might be interesting to combine sequencing results from a different laboratory-reared insect species to see whether the variance of microbial diversity is larger between different life stages of one insect, or between two insect species at the same life stage. Studies on house flies have shown that geographical origin and laboratory rearing can have a considerable impact on the microbiome (Park et al., 2019).

The presence of Wolbachia has been shown to play a role in determining the microbiome composition in Drosophila (Simhadri et al., 2017).

We observed Wolbachia reads in all D. radicum samples regardless of life stage. In the adult stages, the number of reads assigned to this genus ranged from 25 to $65 \%$, which is markedly lower than the $97 \%$ and $80 \%$ reported previously (Bili et al., 2016). The vertical transmission rate of Wolbachia is $100 \%$, and there is no evidence of reproductive manipulation phenotypes such as feminization, parthenogenesis, male-killing or cytoplasmic incompatibility in D. radicum (Lopez et al., 2018). We found particularly high relative abundances of Wolbachia in the pupal samples. One possible explanation for this is that larvae shed their gut lining before pupation, evacuating a large part of the gut-associated bacteria (Hammer and Moran, 2019). The distribution of reads over the nine Wolbachia ASVs suggests that the presence of multiple genotypes of Wolbachia seems unlikely. The artificial OTU richness may stem from technical artifacts such as PCR and/or sequencing errors, or to the limitation of the 16S rRNA gene for taxonomically resolving Wolbachia specifically (Ellegaard et al., 2013). The age-dependency of the relative abundance of Wolbachia in the gut of the termite Nasutitermes arborum was recently described for the first time (Diouf et al., 2018). The relative abundance of Wolbachia 
was shown to be negatively correlated with alpha diversity, suggesting a mutual exclusion from the same environment. Although our sampling methods included homogenization of whole insects, previous studies have indicated that communities from such samples can closely resemble communities that were sampled from the gut alone (Hammer et al., 2014). We therefore suggest that the removal of Wolbachia reads in silico resulted in a dataset that is a closer representation of the gut lumen of $D$. radicum. For future microbiome analyzes of Wolbachia-positive $D$. radicum samples one should consider a more cost-effective approach for reducing the number of Wolbachia reads in a sample by specific restriction digestion of the Wolbachia 16S rRNA gene, as was recently done in Drosophila (Simhadri et al., 2017).

Whether the taxa reported in this study are transient or resident, or even dead or alive, cannot be resolved by the methods presented in this work. Future studies could elucidate the potential of nutritional mutualisms between $D$. radicum and resident microbes.

This work has enabled a deeper understanding of the bacterial players associated with $D$. radicum at different life stages, and of the bacterial nature of the plant rot that is associated with D. radicum infestation. As such, it could potentially provide new clues on symbiotic bacteria that could be exploited in biocontrol programs. Elucidation of the transmission patterns and the specific functions of these bacterial players species are interesting platforms for further research.

\section{DATA AVAILABILITY STATEMENT}

The raw data supporting the conclusions of this article are available on NCBI under the BioProject PRJNA573643.

\section{REFERENCES}

Adam, E., Müller, H., Erlacher, A., and Berg, G. (2016). Complete genome sequences of the Serratia plymuthica strains 3Rp8 and 3Re4-18, two rhizosphere bacteria with antagonistic activity towards fungal phytopathogens and plant growth promoting abilities. Stand. Genomic Sci. 11:61. doi: 10.1186/s40793016-0185-3

Andongma, A. A., Wan, L., Dong, Y.-C., Li, P., Desneux, N., White, J. A., et al. (2015). Pyrosequencing reveals a shift in symbiotic bacteria populations across life stages of Bactrocera dorsalis. Sci. Rep. 5:9470. doi: 10.1038/srep09470

Basset, A., Khush, R. S., Braun, A., Gardan, L., Boccard, F., Hoffmann, J. A., et al. (2000). The phytopathogenic bacteria Erwinia carotovora infects Drosophila and activates an immune response. Proc. Natl. Acad. Sci. U.S.A. 97, 3376-3381. doi: 10.1073/pnas.070357597

Bili, M., Cortesero, A. M., Mougel, C., Gauthier, J. P., Ermel, G., Simon, J. C., et al. (2016). Bacterial community diversity harboured by interacting species. PLoS One 11:e0155392. doi: 10.1371/journal.pone.0155392

Blum, J. E., Fischer, C. N., Miles, J., and Handelsman, J. (2013). Frequent replenishment sustains the beneficial microbiome of Drosophila melanogaster. mBio 4:e00860-13. doi: 10.1128/mBio.00860- 13

Buchner, P. (1965). Endosymbiosis of Animals with Plant Microorganisms. New York, NY: Wiley \& Sons.

Callahan, B. J., Mcmurdie, P. J., and Holmes, S. P. (2017). Exact sequence variants should replace operational taxonomic units in marker-gene data analysis. ISME J. 11, 2639-2643. doi: 10.1038/ismej.2017.119

Callahan, B. J., McMurdie, P. J., Rosen, M. J., Han, A. W., Johnson, A. J. A., and Holmes, S. P. (2016). DADA2: high-resolution sample inference from illumina amplicon data. Nat. Methods 13, 581-583. doi: 10.1038/nmeth.3869
Additional data such as the code for generating figures will be made available, without undue reservation, to any qualified researcher.

\section{AUTHOR CONTRIBUTIONS}

TB and CW designed the research. TB conducted the research, analyzed the data, and wrote the manuscript with input from CW.

\section{FUNDING}

The Nederlandse Organisatie voor Wetenschappelijk Onderzoek (NWO) supported this work through SIAM Gravitation grant 024.002.002. The funder had no role in the design of the study.

\section{ACKNOWLEDGMENTS}

We would like to thank Jeroen Frank and Eric Hester for bioinformatics support and fruitful discussions.

\section{SUPPLEMENTARY MATERIAL}

The Supplementary Material for this article can be found online at: https://www.frontiersin.org/articles/10.3389/fmicb. 2020.00315/full\#supplementary-material

Corby-Harris, V., Pontaroli, A. C., Shimkets, L. J., Bennetzen, J. L., Habel, K. E., and Promislow, D. E. L. (2007). Geographical distribution and diversity of bacteria associated with natural populations of Drosophila melanogaster. Appl. Environ. Microbiol. 73, 3470-3479. doi: 10.1128/AEM.02120-06

Cox, C., and Gilmore, M. (2007). Native microbial colonization of Drosophila melanogaster and its use as a model of Enterococcus faecalis pathogenesis. Infect. Immun. 75, 1565-1576. doi: 10.1128/IAI.01496-06

Diouf, M., Miambi, E., Mora, P., Frechault, S., Robert, A., Rouland-Lefèvre, C., et al. (2018). Variations in the relative abundance of Wolbachia in the gut of Nasutitermes arborum across life stages and castes. FEMS Microbiol. Lett. 365:fny046. doi: 10.1093/femsle/fny046

Dosdall, L. M., Harker, K. N., O’Donovan, J. T., Blackshaw, R. E., Kutcher, H. R., Gan, Y., et al. (2012). Crop sequence effects on root maggot (Diptera: Anthomyiidae: Delia spp.) infestations in canola. J. Econ. Entomol. 105, 12611267. doi: $10.1603 /$ ec11440

Ellegaard, K. M., Klasson, L., Näslund, K., Bourtzis, K., and Andersson, S. G. (2013). Comparative genomics of Wolbachia and the bacterial species concept. PLoS Genet. 9:e1003381. doi: 10.1371/journal.pgen.1003381

Erban, T., Ledvinka, O., Kamler, M., Nesvorna, M., Hortova, B., Tyl, J., et al. (2017). Honeybee (Apis mellifera)-associated bacterial community affected by American foulbrood: detection of Paenibacillus larvae via microbiome analysis. Sci. Rep. 7:5084. doi: 10.1038/s41598-017-05076-8

Geib, S. M., del Mar Jimenez-Gasco, M., Carlson, J. E., Tien, M., Jabbour, R., and Hoover, K. (2009). Microbial community profiling to investigate transmission of bacteria between life stages of the wood-boring beetle, Anoplophora glabripennis. Microb. Ecol. 58, 199-211. doi: 10.1007/s00248-009-9501-4

Gupta, A. K., Nayduch, D., Verma, P., Shah, B., Ghate, H. V., Patole, M. S., et al. (2012). Phylogenetic characterization of bacteria in the gut of house flies (Musca 
domestica L.). FEMS Microbiol. Ecol. 79, 581-593. doi: 10.1111/j.1574-6941. 2011.01248.x

Hammer, T. J., Janzen, D. H., Hallwachs, W., Jaffe, S. L., and Fierer, N. (2017). Caterpillars lack a resident gut microbiome. Proc. Natl. Acad. Sci. U.S.A. 114, 9641-9646. doi: 10.1101/132522

Hammer, T. J., McMillan, W. O., and Fierer, N. (2014). Metamorphosis of a butterfly-associated bacterial community. PLoS One 9:e86995. doi: 10.1371/ journal.pone.0086995

Hammer, T. J., and Moran, N. A. (2019). Links between metamorphosis and symbiosis in holometabolous insects. Philos. Trans. R. Soc. B Biol. Sci. 374:20190068. doi: 10.1098/rstb.2019.0068

Kikuchi, Y., Hayatsu, M., Hosokawa, T., Nagayama, A., Tago, K., and Fukatsu, T. (2012). Symbiont-mediated insecticide resistance. Proc. Natl. Acad. Sci. U.S.A. 109, 8618-8622. doi: 10.1073/Pnas.1200231109

Kim, B. R., Shin, J., Guevarra, R. B., Lee, J. H., Kim, D. W., Seol, K. H., et al. (2017). Deciphering diversity indices for a better understanding of microbial communities. J. Microbiol. Biotechnol. 27, 2089-2093. doi: 10.4014/jmb.1709. 09027

Klindworth, A., Pruesse, E., Schweer, T., Peplies, J., Quast, C., Horn, M., et al. (2013). Evaluation of general 16S ribosomal RNA gene PCR primers for classical and next-generation sequencing-based diversity studies. Nucleic Acids Res. 41:e1. doi: 10.1093/nar/gks808

Kwong, W. K., Medina, L. A., Koch, H., Sing, K. W., Soh, E. J. Y., Ascher, J. S., et al. (2017). Dynamic microbiome evolution in social bees. Sci. Adv. 3:e1600513. doi: $10.1126 /$ sciadv. 1600513

Lopez, V., Cortesero, A. M., and Poinsot, D. (2018). Influence of the symbiont Wolbachia on life history traits of the cabbage root fly (Delia radicum). J. Invertebr. Pathol. 158, 24-31. doi: 10.1016/j.jip.2018. 09.002

Lukwinski, A. T., Hill, J. E., Khachatourians, G. G., Hemmingsen, S. M., and Hegedus, D. D. (2006). Biochemical and taxonomic characterization of bacteria associated with the crucifer root maggot (Delia radicum). Can. J. Microbiol. 52, 197-208. doi: 10.1139/w05-123

Martinez Arbizu, P. (2019). pairwiseAdonis: Pairwise Multilevel Comparison Using Adonis. R Package Version 0.3. Available online at: https://github.com/ pmartinezarbizu/pairwiseAdonis (accessed April 29, 2019).

Mason, C. J., Jones, A. G., and Felton, G. W. (2018). Co-option of microbial associates by insects and their impact on plant-folivore interactions. Plant Cell Environ. 42, 1078-1086. doi: 10.1111/pce.13430

McMurdie, P. J., and Holmes, S. (2013). Phyloseq: an R package for reproducible interactive analysis and graphics of microbiome census data. PLoS One 8:e61217. doi: 10.1371/journal.pone.0061217

Oksanen, J., Blanchet, F. G., Friendly, M., Kindt, R., Legendre, P., McGlinn, D., et al. (2019). vegan: Community Ecology Package. Available online at: https: //cran.r-project.org/package=vegan (accessed April 29, 2019).

Oliver, K. M., Moran, N. A., and Hunter, M. S. (2005). Variation in resistance to parasitism in aphids is due to symbionts not host genotype. Proc. Natl. Acad. Sci. U.S.A. 102, 12795-12800. doi: 10.1073/pnas.050613 1102

Park, D. S., Oh, H. W., Jeong, W. J., Kim, H., Park, H. Y., and Bae, K. S. (2007). A culture-based study of the bacterial communities within the guts of nine longicorn beetle species and their exo-enzyme producing properties for degrading xylan and pectin. J. Microbiol. 45, 394-401.

Park, R., Dzialo, M. C., Spaepen, S., Nsabimana, D., Gielens, K., Devriese, H., et al. (2019). Microbial communities of the house fly Musca domestica vary with geographical location and habitat. Microbiome 7:147. doi: 10.1186/s40168-0190748-9

Salas, B., Conway, H. E., Schuenzel, E. L., Hopperstad, K., Vitek, C., and Vacek, D. C. (2017). Morganella morganii (Enterobacteriales: Enterobacteriaceae) is a lethal pathogen of Mexican fruit fly (Diptera: Tephritidae) larvae. Florida Entomol. 100, 743-751. doi: 10.1653/024.100.0422

Serbus, L. R., Casper-Lindley, C., Landmann, F., and Sullivan, W. (2008). The genetics and cell biology of Wolbachia-host interactions. Annu. Rev. Genet. 42, 683-707. doi: 10.1146/annurev.genet.41.110306.130354

Simhadri, R. K., Fast, E. M., Guo, R., Schultz, M. J., Vaisman, N., Ortiz, L., et al. (2017). The gut commensal microbiome of Drosophila melanogaster is modified by the endosymbiont Wolbachia. mSphere 2:e00287-17. doi: 10.1128/mSphere. 00287- 17

Smith, K. M. (1927). A study of Hylemyia (Chortophila) brassicae Bouche, the cabbage root fly and its parasites. With notes on some other Dipterous pests of cruciferous plants. Ann. Appl. Biol. 14, 312-330. doi: 10.1111/j.1744-7348. 1927.tb07015.x

Somerville, J., Zhou, L., and Raymond, B. (2019). Aseptic rearing and infection with gut bacteria improve the fitness of transgenic diamondback moth, Plutella xylostella. Insects 10:89. doi: 10.3390/insects 10040089

Soroka, J. J., Dosdall, L. M., Olfert, O. O., and Seidle, E. (2004). Root maggots (Delia spp., Diptera: Anthomyiidae) in prairie canola (Brassica napus L. and B. rapa L.): spatial and temporal surveys of root damage and prediction of damage levels. Can. J. Plant Sci. 84, 1171-1182. doi: 10.4141/P02-174

Stamatakis, A. (2006). RAxML-VI-HPC: maximum likelihood-based phylogenetic analyses with thousands of taxa and mixed models. Bioinformatics 22, 26882690. doi: 10.1093/bioinformatics/btl446

Tierens, K. F., Thomma, B. P., Brouwer, M., Schmidt, J., Kistner, K., Porzel, A., et al. (2001). Study of the role of antimicrobial glucosinolate-derived isothiocyanates in resistance of Arabidopsis to microbial pathogens. Plant Physiol. 125, 1688 1699. doi: 10.1104/pp.125.4.1688

van den Bosch, T. J. M., Outi, N., and Welte, C. U. (2019). Single gene enables plant pathogenic Pectobacterium to overcome host-specific chemical defence. Mol. Plant. Pathol. (in press). doi: 10.1111/mpp.12900

Van den Bosch, T. J. M., Tan, K., Joachimiak, A., and Welte, C. U. (2018). Functional profiling and crystal structures of isothiocyanate hydrolases found in gut-associated and plant pathogenic bacteria. Appl. Environ. Microbiol. 84, 1-18. doi: 10.1128/AEM.00478-18

Welte, C. U., de Graaf, R. M., van den Bosch, T. J. M., Op den Camp, H. J. M., van Dam, N. M., and Jetten, M. S. M. (2016a). Plasmids from the gut microbiome of cabbage root fly larvae encode SaxA that catalyses the conversion of the plant toxin 2-phenylethyl isothiocyanate. Environ. Microbiol. 18, 1379-1390. doi: 10.1111/1462-2920.12997

Welte, C. U., Rosengarten, J. F., de Graaf, R. M., and Jetten, M. S. M. (2016b). SaxAmediated isothiocyanate metabolism in phytopathogenic pectobacteria. Appl. Environ. Microbiol. 82, 2372-2379. doi: 10.1128/AEM.04054-15

Yilmaz, P., Parfrey, L. W., Yarza, P., Gerken, J., Pruesse, E., Quast, C., et al. (2014). The SILVA and "all-species Living Tree Project (LTP)" taxonomic frameworks. Nucleic Acids Res. 42, 643-648. doi: 10.1093/nar/gkt1209

Zhao, Y., Wang, W., Zhu, F., Wang, X., Wang, X., Lei, C., et al. (2017). The gut microbiota in larvae of the housefly Musca domestica and their horizontal transfer through feeding. AMB Expr. 7:147. doi: 10.1186/s13568-017-0445-7

Conflict of Interest: The authors declare that the research was conducted in the absence of any commercial or financial relationships that could be construed as a potential conflict of interest.

Copyright (c) 2020 van den Bosch and Welte. This is an open-access article distributed under the terms of the Creative Commons Attribution License (CC BY). The use, distribution or reproduction in other forums is permitted, provided the original author(s) and the copyright owner(s) are credited and that the original publication in this journal is cited, in accordance with accepted academic practice. No use, distribution or reproduction is permitted which does not comply with these terms. 